TRANSACTIONS OF THE

AMERICAN MATHEMATICAL SOCIETY

Volume 351, Number 7, Pages 2949-2960

$\mathrm{S}$ 0002-9947(99)02365-X

Article electronically published on March 8, 1999

\title{
COMPRESSIONS OF RESOLVENTS AND MAXIMAL RADIUS OF REGULARITY
}

\author{
C. BADEA AND M. MBEKHTA
}

\begin{abstract}
Suppose that $\lambda-T$ is left invertible in $L(H)$ for all $\lambda \in \Omega$, where $\Omega$ is an open subset of the complex plane. Then an operator-valued function $L(\lambda)$ is a left resolvent of $T$ in $\Omega$ if and only if $T$ has an extension $\tilde{T}$, the resolvent of which is a dilation of $L(\lambda)$ of a particular form. Generalized resolvents exist on every open set $U$, with $\bar{U}$ included in the regular domain of $T$. This implies a formula for the maximal radius of regularity of $T$ in terms of the spectral radius of its generalized inverses. A solution to an open problem raised by J. Zemánek is obtained.
\end{abstract}

\section{INTRODUCTION}

Let $H$ be a complex, separable, infinite dimensional Hilbert space and let $L(H)$ be the $\mathrm{C}^{*}$-algebra of all continuous linear operators on $H$. The resolvent set $\rho(T)$ of $T \in L(H)$ is, by definition, the set of all complex numbers $\lambda \in \mathbb{C}$ such that the operator $\lambda I-T$ is invertible in the algebra $L(H)$. Then its resolvent

$$
R(\lambda)=(\lambda I-T)^{-1}
$$

is an analytic function on $\rho(T)$ such that

$$
R(\lambda)(\lambda I-T)=(\lambda I-T) R(\lambda)=I
$$

for all $\lambda \in \rho(T)$. Here $I$ is the identity operator on $H$. Moreover, $R(\lambda)$ satisfies the first resolvent equation, namely

$$
R(\lambda)-R(\mu)=(\mu-\lambda) R(\lambda) R(\mu)
$$

for all $\lambda, \mu \in \rho(T)$. The spectrum $\sigma(T)$ of $T$ is the complement of $\rho(T)$ in $\mathbb{C}$. The spectral radius $r_{\sigma}(T)$ of $T$ is

$$
r_{\sigma}(T)=\sup \{|\lambda|: \lambda \in \sigma(T)\} .
$$

The left resolvent set $\rho_{\ell}(T)$ of the bounded linear operator $T$ is defined as the set of all complex numbers $\lambda \in \mathbb{C}$ such that $\lambda I-T$ is left invertible in $L(H)$, that is, oneto-one and with closed range. The left spectrum of $T$ is the set $\sigma_{\ell}(T)=\mathbb{C} \backslash \rho_{\ell}(T)$. According to a result due to G. R. Allan $[1,2]$, there exists an analytic operatorvalued function $L(\lambda)$ on $\rho_{\ell}(T)$ such that $L(\lambda)(\lambda I-T)=I$, for all $\lambda \in \rho_{\ell}(T)$. An operator mapping $L(\lambda): U \rightarrow L(H), U \subseteq \rho_{\ell}(T)$, is said to be a left resolvent of $T$ on $U$, if $L(\lambda)(\lambda I-T)=I$ on $U$ and, in addition, $L(\lambda)$ satisfies the resolvent equation above for all $\lambda$ and $\mu$ in the same connected component of $U$. If $U=\rho_{\ell}(T)$,

Received by the editors February 17, 1997.

1991 Mathematics Subject Classification. Primary 47A10, 47A20.

Key words and phrases. One-sided resolvents, Hilbert space operators, spectral radius, dilations and compressions. 
the above $L(\lambda)$ is simply called a left resolvent of $T$. The right resolvent set $\rho_{r}(T)$ and right resolvents are defined in a similar way. Note that the resolvent identity implies the analyticity of the operator valued function.

Let $\operatorname{reg}(T)$ denote the sets of all complex numbers $\lambda$ for which $\mu I-T$ possess analytic generalized inverses, for all $\mu$ in a neighborhood of $\lambda$; that is, there exist $U_{\lambda}$, a neighborhood of $\lambda$, and an operator-valued analytic function $G(\mu): U_{\lambda} \rightarrow L(H)$ such that, for all $\mu \in U_{\lambda}$,

$$
(\mu I-T) G(\mu)(\mu I-T)=(\mu I-T)
$$

and

$$
G(\mu)(\mu I-T) G(\mu)=G(\mu)
$$

Then $\sigma_{g}(T)=\mathbb{C} \backslash \operatorname{reg}(T)$ is the generalized spectrum of $T$ [11], [12]. Note also that $\operatorname{reg}(T)$ has several equivalent definitions. According to a result of Shubin [17], there exists a global analytic function $G(\lambda)$ defined on $\operatorname{reg}(T)$ which is a generalized inverse of $\lambda I-T$ for all $\lambda \in \operatorname{reg}(T)$. By definition, a generalized resolvent of $T$ on $U, U \subseteq \operatorname{reg}(T)$, is an operator-valued mapping $G(\lambda)$ satisfying the conditions (1.1) and (1.2) for $\mu \in U$ and the resolvent equation for all $\lambda$ and $\mu$ in the same connected component of $U$. Generalized resolvents of $T$ are generalized resolvents of $T$ on $\operatorname{reg}(T)$. If $U \subseteq \rho(T)$, then a generalized (left, right) resolvent on $U$ is unique and coincides with the usual resolvent.

It is not known if left or generalized resolvents always exist. These open problems go back to C. Apostol and K. Clancey [4], [5] and P. Saphar [15], [16]. See also the list of references in [7] for several contributions on these problems.

The aim of the present paper is twofold. Firstly, we will prove that generalized resolvents for $T$ always exist on every open subset $U$ of $\operatorname{reg}(T)$ such that $\bar{U} \subset$ $\operatorname{reg}(T)$. This implies a formula for the maximal radius of regularity of $T$ in terms of the spectral radius of its generalized inverses. In particular, one obtains (section 2), in the case of Hilbert space operators, a positive answer to a problem recently raised by Zemánek [19] for Banach algebra elements. Note that in [7] several partial answers to Zemánek's question (for closed operators with a dense domain on a Banach space) were given.

Secondly, we give a new characterization of left resolvents in terms of dilations and compressions. The result of C.J. Read [14], about the extent to which an extension of a Hilbert space operator reduces the spectrum, implies that every operator $T$ admits an extension $\tilde{T}$ to a larger Hilbert space $\tilde{H}=H \oplus H^{\prime}$ such that $\lambda I-\tilde{T}$ is invertible in $L(\tilde{H})$ for all $\lambda \in \rho_{\ell}(T)$. Moreover, an analytic left inverse function $L(\lambda)$ of $\lambda I-T$ can be obtained as the compression on $H$ of $R(\tilde{T})(\lambda)=$ $(\lambda I-\tilde{T})^{-1}$. The matrix of $R(\tilde{T})(\lambda)$ with respect to the decomposition $\tilde{H}=H \oplus H^{\prime}$ will then be of the form

$$
\left(\begin{array}{cc}
L(\lambda) & * \\
* & *
\end{array}\right)
$$

where $*$ means a suitable entry.

The main result of the second part of this note is to prove that $L(\lambda)$ is a left resolvent of $T$ in $\Omega \subseteq \rho_{\ell}(T) \cap \sigma(T)$ if and only if there exists an extension $\tilde{T}$ of $T$ on a larger Hilbert space $\tilde{H}=H \oplus H^{\prime}$ such that $R(\tilde{T})(\lambda)=(\lambda I-\tilde{T})^{-1}$ is invertible in $L(\tilde{H})$ for all $\lambda \in \Omega$ and the matrix of $R(\tilde{T})(\lambda)$ with respect to the decomposition 
$\tilde{H}=H \oplus H^{\prime}$ has the form

$$
\left(\begin{array}{cc}
L(\lambda) & 0 \\
* & *
\end{array}\right)
$$

that is, $R(\tilde{T})(\lambda)$ leaves $H^{\prime}$ invariant, for all $\lambda \in \Omega$. In fact, a stronger version of this result will be proved.

The paper is organized as follows. The existence of generalized resolvents and its consequences for the maximal radius of regularity are discussed in Section 2. In the next section the characterization of left resolvents and related results are presented.

The first author wish to thank Dan Timotin for useful discussions concerning Read's theorem. The second author wish to thank University of Galatasaray for its hospitality.

\section{Generalized Resolvents on subsets of $\operatorname{reg}(T)$ AND RADIUS OF REGULARITY}

2.1. Generalized resolvents on subsets of $\operatorname{reg}(T)$. The following result is a partial result for the one-sided resolvent problem.

Lemma 2.1 ([6]). Let $T \in L(H)$ and let $\Omega \subset \bar{\Omega} \subset \rho_{\ell}(T)$ be an open set. Then there is a left resolvent of $T$ on $\Omega$.

Proof. The result follows from Proposition 9.17 [6]; see also Lemma 3.18 [9] and Errata to [9].

The following result is the corresponding extension for generalized resolvents.

Theorem 2.2. Let $T \in L(H)$ and let $\Omega \subset \bar{\Omega} \subset \operatorname{reg}(T)$ be an open set. Then there is a generalized resolvent of $T$ on $\Omega$; that is, there exists an analytic function $G: \Omega \rightarrow L(H)$ such that $G(\lambda)$ satisfies the resolvent identity for all $\lambda$ and $\mu$ in the same connected component of $U$ and

$$
(\lambda I-T) G(\lambda)(\lambda I-T)=(\lambda I-T), \lambda \in \Omega,
$$

as well as

$$
G(\lambda)(\lambda I-T) G(\lambda)=G(\lambda), \quad \lambda \in \Omega .
$$

Proof. Let $H=H_{r} \oplus H_{0} \oplus H_{l}$ be the Apostol decomposition [3], [10]. With respect to this decomposition, the operator $T$ can be written as

$$
\left(\begin{array}{ccc}
T_{r} & A & B \\
0 & T_{0} & C \\
0 & 0 & T_{l}
\end{array}\right)
$$

for suitable operator entries. Then [10, Théorème 4.10]

$$
\operatorname{reg}(T)=\rho_{r}\left(T_{r}\right) \cap \rho\left(T_{0}\right) \cap \rho_{\ell}\left(T_{l}\right) .
$$

Using two times Lemma 2.1, we find a left resolvent $L(\lambda)$ for $T_{l}$ and a right resolvent $R(\lambda)$ for $T_{r}$, both defined on $\Omega \subset \bar{\Omega} \subset \operatorname{reg}(T)$. In particular, $\left(\lambda I-T_{r}\right) R(\lambda)=I$ and $L(\lambda)\left(\lambda I-T_{\ell}\right)=I$ for all $\lambda \in \Omega$. Consider $G: \Omega \rightarrow L(H)$, given by the following matrix, with respect to the Apostol decomposition :

$$
\left(\begin{array}{ccc}
R(\lambda) & R(\lambda) A R\left(T_{0}\right)(\lambda) & R(\lambda)\left[A R\left(T_{0}\right)(\lambda) C+B\right] L(\lambda) \\
0 & R\left(T_{0}\right)(\lambda) & R\left(T_{0}\right)(\lambda) C L(\lambda) \\
0 & 0 & L(\lambda)
\end{array}\right)
$$


where $R\left(T_{0}\right)(\lambda)=\left(\lambda I-T_{0}\right)^{-1}$. Let $P(\lambda)=(\lambda I-T) G(\lambda)$ and $Q(\lambda)=G(\lambda)(\lambda I-T)$ for $\lambda \in \Omega$. Then simple computation shows that

$$
P(\lambda)=\left(\begin{array}{ccc}
I & 0 & 0 \\
0 & I & 0 \\
0 & 0 & \left(\lambda I-T_{\ell}\right) L(\lambda)
\end{array}\right)
$$

and

$$
Q(\lambda)=\left(\begin{array}{ccc}
R(\lambda)\left(\lambda I-T_{r}\right) & 0 & 0 \\
0 & I & 0 \\
0 & 0 & I
\end{array}\right) .
$$

Therefore $G(\lambda)$ is a global generalized inverse for $T$ on $\Omega$. We show now that $G(\lambda)$ satisfies the resolvent identity on $\Omega$. To this end, we use a criterion given in [7, Theorem 2.7]. It is easy to show that the range of $P(\lambda)$ coincides with the range of $\lambda I-T$ and the kernel of $Q(\lambda)$ coincides with the kernel of $\lambda I-T$. On the other hand, we have

$$
P(\lambda) P(\mu)=\left(\begin{array}{ccc}
I & 0 & 0 \\
0 & I & 0 \\
0 & 0 & \left(\lambda I-T_{\ell}\right) L(\lambda)\left(\mu I-T_{\ell}\right) L(\mu)
\end{array}\right) .
$$

But, using the fact that $L(\lambda)$ is a left resolvent of $\lambda I-T_{\ell}(T)$, we obtain

$$
\begin{aligned}
\left(\lambda I-T_{\ell}\right) L(\lambda)\left(\mu I-T_{\ell}\right) L(\mu) & =\left(\lambda I-T_{\ell}\right) L(\lambda)\left(\mu I-\lambda I+\lambda I-T_{\ell}\right) L(\mu) \\
& =\left(\lambda I-T_{\ell}\right)(\mu-\lambda) L(\lambda) L(\mu)+\left(\lambda I-T_{\ell}\right) L(\mu) \\
& =\left(\lambda I-T_{\ell}\right)(L(\lambda)-L(\mu))+\left(\lambda I-T_{\ell}\right) L(\mu) \\
& =\left(\lambda I-T_{\ell}\right) L(\lambda) .
\end{aligned}
$$

Therefore $P(\lambda) P(\mu)=P(\lambda)$ and, similarly, $Q(\lambda) Q(\mu)=Q(\mu)$. Using [7, Proof of Theorem 2.7], we get that $G(\lambda)$ satisfies the resolvent identity in $\Omega$.

2.2. Maximal radius of regularity. As an application of the existence of generalized resolvents, the following formula for the maximal radius of regularity can be proved. It is a generalization of [7, Theorem 3.1]. Denote by

$$
\operatorname{dist}(a, A)=\inf \{|a-z|: z \in A\}
$$

the distance from $a \in \mathbb{C}$ to the set $A \subset \mathbb{C}$.

Theorem 2.3. Let $T \in L(H)$ be a linear operator such that $0 \in \operatorname{reg}(T)$. Then

$$
\operatorname{dist}\left(0, \sigma_{g}(T)\right)=\sup \left\{\frac{1}{r_{\sigma}(S)}: T S T=T\right\},
$$

where $r_{\sigma}(S)$ is the spectral radius of $S$.

Proof. The proof is similar to that of [7, Theorem 3.1], where the present theorem has been proved (for closed, densely defined, Banach space operators) under the additional assumption that $T$ is Fredholm. Therefore some details will be omitted. Let $S \in L(H)$ be a generalized inverse of $T$. Then, using for instance [7, Theorem 2.4], we get $T^{n} S^{n} T^{n}=T^{n}$, for all $n \geq 1$. Let $\gamma(T)$ be the reduced minimum modulus of $T$ :

$$
\gamma(T)=\inf \{\|T x\|: \operatorname{dist}(x, N(T))=1\},
$$


where $N(T)$ is the kernel of $T$. By [12, Lemme 3.5], we have $\gamma\left(T^{n}\right) \geq 1 /\left(\left\|S^{n}\right\|\right)$ and therefore

$$
\lim _{n \rightarrow \infty} \gamma\left(T^{n}\right)^{1 / n} \geq \frac{1}{r_{\sigma}(S)}
$$

Using [12, Théorème 3.1] we get the inequality

$$
\operatorname{dist}\left(0, \sigma_{g}(T)\right) \geq \sup \left\{\frac{1}{r_{\sigma}(S)}: T S T=T\right\} .
$$

In order to prove the other inequality, set $d=\operatorname{dist}\left(0, \sigma_{g}(T)\right)$. Then

$$
B(0, d)=\{\lambda \in \mathbb{C}:|\lambda|<d\} \subseteq \operatorname{reg}(T) .
$$

Let $\varepsilon$ be a positive number and put

$$
U=B\left(0, \frac{d}{1+\varepsilon}\right)=\left\{\lambda \in \mathbb{C}:|\lambda|<\frac{d}{1+\varepsilon}\right\} .
$$

Then $U \subset \bar{U} \subset \operatorname{reg}(T)$. Using Theorem 2.2, there is a generalized resolvent $G(\lambda)$ for $T$ on $U$. Then, for all $\lambda \in U$,

$$
G(\lambda)=\sum_{n=0}^{\infty} \lambda^{n} T_{n}, \quad T_{n} \in L(H) .
$$

As in [7, Proof of Theorem 3.1], we can use the Cauchy's integral formula and [7, Theorem 2.6] to get

$$
\left\|T_{n}\right\| \leq M\left(\frac{1+\varepsilon}{d}\right)^{n+1}, \quad n \geq 0
$$

where $M=\max \{\|G(\lambda)\|: \lambda \in \bar{U}\}$, and $T_{n}=(-1)^{n+1} T_{0}^{n+1}$. Thus

$$
\left\|T_{0}^{n+1}\right\| \leq M\left(\frac{1+\varepsilon}{d}\right)^{n+1}, \quad n \geq 0
$$

which implies $r_{\sigma}\left(T_{0}\right) \leq(1+\varepsilon) / d$. Since $T T_{0} T=T$, we have

$$
\sup \left\{\frac{1}{r_{\sigma}(S)}: T S T=T\right\} \geq \frac{1}{r_{\sigma}\left(T_{0}\right)} \geq \frac{d}{1+\varepsilon} .
$$

Since $\varepsilon>0$ was arbitrarily chosen, we have

$$
\sup \left\{\frac{1}{r_{\sigma}(S)}: T S T=T\right\} \geq d .
$$

The proof is now complete.

Remark 2.4. The above proof shows that the sup is attained (for some $S_{0}$ ) in the formula

$$
d:=\operatorname{dist}\left(0, \sigma_{g}(T)\right)=\sup \left\{\frac{1}{r_{\sigma}(S)}: T S T=T\right\},
$$

if one is able to construct a generalized resolvent for $T$ on $B(0, d)$. Conversely, if there exists $S_{0} \in L(H)$ such that $T S_{0} T=T$ and $d=\frac{1}{r_{\sigma}\left(S_{0}\right)}$, then $G(\lambda)=$ $\sum_{n=0}^{\infty} \lambda^{n} S_{0}^{n+1}$ is a generalized resolvent for $T$ on $B(0, d)$. We omit here the details.

Set $s(T)=\sup \left\{\frac{1}{r_{\sigma}(S)}: T S T=T\right\}$.

Corollary 2.5. Let $T \in L(H)$ and suppose that $0 \in \operatorname{reg}(T)$. Then we have $s\left(T^{n}\right)=s(T)^{n}$, for all $n \geq 1$. 
Proof. Let $n \geq 1$. Using the preceding theorem and [12, Theorem 3.1], we get

$$
s(T)=\lim _{k \rightarrow \infty} \gamma\left(T^{k}\right)^{1 / k} .
$$

Therefore

$$
s\left(T^{n}\right)=\lim _{k \rightarrow \infty}\left(\gamma\left(T^{k n}\right)^{1 / k n}\right)^{n}=s(T)^{n} .
$$

The proof is complete.

The following is a solution for a problem raised by J. Zemánek [19] in the more general setting of Banach algebras.

Corollary 2.6. Let $T \in L(H)$ be a linear operator such that $0 \in \rho_{\ell}(T)$. Then

$$
\operatorname{dist}\left(0, \sigma_{\ell}(T)\right)=\sup \left\{\frac{1}{r_{\sigma}(S)}: S T=I\right\} .
$$

Proof. The result follows from Theorem 2.3 and is similar to the proof of Corollary 3.4 in [7].

Corollary 2.6 can be viewed as a one-sided generalization of the known formula

$$
0 \in \rho(T) \quad \Longrightarrow \quad \operatorname{dist}(0, \sigma(T))=\frac{1}{r_{\sigma}\left(T^{-1}\right)} .
$$

\section{LEFT RESOLVENTS AS COMPRESSIONS OF RESOLVENTS}

3.1. Read's extension theorem. The following result is a consequence of a result of Read [14] and Corollary 2.6.

Lemma 3.1. Let $T \in L(H)$ and $\Omega \subseteq \rho_{\ell}(T)$. There exist a larger Hilbert space $\tilde{H}=H \oplus H^{\prime}$ containing $H$ and an extension $\tilde{T}$ of $T$ on $\tilde{H}$ such that $\lambda I-\tilde{T}$ is invertible in $L(\tilde{H})$ for all $\lambda \in \Omega$. Moreover,

$$
r_{\sigma}\left((\lambda I-\tilde{T})^{-1}\right)=\inf \left\{r_{\sigma}(S): S(T-\lambda I)=I\right\}
$$

for all $\lambda \in \Omega$.

Proof. According to a result due to Read [14], there exist a larger Hilbert space $\tilde{H}=H \oplus H^{\prime}$ and an extension $\tilde{T}$ of $T$ such that the spectrum $\sigma(\tilde{T})$ of $\tilde{T}$ is equal to the approximate point spectrum $\sigma_{a p}(T)$ of $T$, that is

$$
\sigma(\tilde{T})=\sigma_{a p}(T):=\{\lambda \in \mathbb{C}: \inf \{\|(\lambda I-T) x\|: x \in H,\|x\|=1\}=0\} .
$$

This coincides with the left spectrum $\sigma_{\ell}(T)$ for Hilbert space operators.

For the second part, using Corollary 2.6, we have

$$
\begin{aligned}
\frac{1}{r_{\sigma}\left((\lambda I-\tilde{T})^{-1}\right)} & =\operatorname{dist}(\lambda, \sigma(\tilde{T})) \\
& =\operatorname{dist}\left(\lambda, \sigma_{\ell}(T)\right) \\
& =\sup \left\{\frac{1}{r_{\sigma}(S)}: S(T-\lambda I)=I\right\} .
\end{aligned}
$$

This implies the above equality. 
Remark 3.2. In Read's theorem [14], $H^{\prime}$ can be chosen as a copy of $H$ and the norm of $\tilde{T}$ almost the norm of $T$; that is, if $\varepsilon>0, \tilde{T}$ (and $H^{\prime} \cong H$ ) can be chosen such that $\|\tilde{T}\| \leq(1+\varepsilon)\|T\|$. We also want to note that $H^{\prime} \cong H$ depends upon $T$ in a sensitive way. Indeed, consider an analytic, operator-valued function $\Omega \ni \lambda \rightarrow T(\lambda) \in L(H)$. By Read's proof, it is possible to find a copy $H^{\prime}(\lambda)$ of $H$ and extensions $\Omega \ni \lambda \rightarrow \tilde{T}(\lambda) \in L\left(H \oplus H^{\prime}(\lambda)\right)$ such that $\sigma(\tilde{T}(\lambda))=\sigma_{\ell}(T(\lambda))$. However, there are examples where one cannot find a universal copy of $H$ in place of $H^{\prime}(\lambda)$ and analytic family $\Omega \ni \lambda \rightarrow \tilde{T}(\lambda) \in L\left(H \oplus H^{\prime}\right)$ of extensions. We use for this purpose an example of Ransford [13]. Consider $\Omega=\mathbb{C}, H=\ell^{2}$ and

$$
T(\lambda)\left(a_{1}, a_{2}, \ldots\right)=\left(a_{1}, \lambda a_{1}, a_{2}, \lambda a_{2}, \ldots\right)
$$

which is holomorphic. Then it can be shown that

$$
\sigma_{\ell}(T(\lambda)) \subseteq\left\{z:|z|=\left(1+|\lambda|^{2}\right)^{1 / 2}\right\} .
$$

Suppose that an analytic family $\tilde{T}(\lambda) \in L(H \oplus H)$, extensions of $T(\lambda)$, such that $\sigma(\tilde{T}(\lambda))=\sigma_{\ell}(T(\lambda))$ would exist. Then $\lambda \rightarrow \sigma_{\ell}(T(\lambda))$ would be an analytic setvalued function as the spectrum of an analytic family of operators (cf. [18] for definitions and properties of set-valued analytic functions). This is in contradiction with the fact that

$$
\phi(\lambda)=\sup \left\{-\log |z|: z \in \mathbb{C} \backslash \rho_{\ell}(T(\lambda))\right\}=-\log \left(1+|\lambda|^{2}\right)^{1 / 2}
$$

is not subharmonic on $\mathbb{C}$ (cf. [13]). Indeed, $\phi$ attains a maximum at 0 .

3.2. Compressions of resolvents. Lemma 3.1 implies Allan's result mentioned in the Introduction. Indeed, let

$$
L(\lambda) h=P_{H} R(\tilde{T})(\lambda) h, \quad h \in H,
$$

be the compression on $H$ of $R(\tilde{T})(\lambda)=(\lambda I-\tilde{T})^{-1}$. Then, using the equality $R(\tilde{T})(\lambda)(\lambda I-\tilde{T})=I$ and the fact that $\tilde{T}$ is an extension on $\tilde{H}$ of $T$, we obtain

$$
L(\lambda)(\lambda I-T)=I \text { on } H \text {. }
$$

Since $L(\lambda)$ is analytic, we obtain a global, analytic left inverse function of $\lambda I-T$, i.e. Allan's result. The matrix of $R(\tilde{T})(\lambda)$ with respect to the decomposition $\tilde{H}=$ $H \oplus H^{\prime}$ will then be of the form

$$
\left(\begin{array}{cc}
L(\lambda) & * \\
* & *
\end{array}\right)
$$

The following characterization of left resolvents is the main result of this section.

Theorem 3.3. Let $T \in L(H)$ and let $\Omega$ be an open, connected subset of $\rho_{\ell}(T) \cap$ $\sigma(T)$. Then $L(\lambda)$ is a left resolvent of $T$ in $\Omega$ if and only if there exists an extension $\tilde{T}$ of $T$ on a larger Hilbert space $\tilde{H}=H \oplus H^{\prime}$ such that $R(\tilde{T})(\lambda)=(\lambda I-\tilde{T})^{-1}$ exists in $L(\tilde{H})$ for all $\lambda \in \Omega$ and the matrix of $R(\tilde{T})(\lambda)$ with respect to the decomposition $\tilde{H}=H \oplus H^{\prime}$ has the form

$$
\left(\begin{array}{cc}
L(\lambda) & 0 \\
T(\lambda) & V(\lambda)
\end{array}\right)
$$

with suitable operator-valued functions $T(\lambda)$ and $V(\lambda)$. 
The condition $\Omega \subset \sigma(T)$ is explained by the fact that on $\Omega \cap \rho(T)$ the usual resolvent is also a left resolvent. The condition of connectedness of $\Omega$ is justified by the definition of left resolvents as left inverses satisfying the resolvent identity on every connected component.

For the " only if" part of Theorem 3.3, the following stronger result can be proved.

Theorem 3.4. Let $T \in L(H)$ and let $\Omega \subset \rho_{\ell}(T) \cap \sigma(T)$ be an open, connected subset. Suppose that there exists an extension $\tilde{T}$ of $T$ on a larger Hilbert space $\tilde{H}=H \oplus H^{\prime}$ such that $\lambda I-\tilde{T}$ is invertible in $L(\tilde{H})$ for all $\lambda \in \Omega$ and the matrix of $R(\tilde{T})(\lambda)=(\lambda I-\tilde{T})^{-1}$ with respect to the decomposition $\tilde{H}=H \oplus H^{\prime}$ has the form

$$
\left(\begin{array}{ll}
L(\lambda) & S(\lambda) \\
T(\lambda) & V(\lambda)
\end{array}\right)
$$

for some operator-valued functions $L(\lambda), S(\lambda)$ defined on $H$ and $H^{\prime}$, respectively, with values in $H$ and $T(\lambda), V(\lambda)$ defined on $H$ and $H^{\prime}$, respectively, with values in $H^{\prime}$. Suppose that $S(\lambda) T(\lambda)=0$ for all $\lambda \in \Omega$. Then $L(\lambda)$ is a left resolvent of $T$ in $\Omega$.

Clearly, the above condition is satisfied if $S(\lambda) \equiv 0$.

For the "if" part of Theorem 3.3, we need a construction of independent interest. Namely, we will prove the following result.

Theorem 3.5. Suppose that $T \in L(H)$ and let $\Omega$ be an open, connected subset of $\rho_{\ell}(T) \cap \sigma(T)$. Let $L(\lambda)$ be a global, analytic left-inverse function of $T-\lambda I$. There exist a larger Hilbert space $\tilde{H}=H \oplus H^{\prime}$ and a family of operators $\tilde{T}(\lambda) \in L(\tilde{H}), \lambda \in$ $\Omega$, such that

1. $\tilde{T}(\lambda)$ is an extension of $T$, for all $\lambda \in \Omega$;

2. $\lambda I-\tilde{T}(\lambda)$ is invertible, for all $\lambda \in \Omega$;

3. With respect to the decomposition $\tilde{H}=H \oplus H^{\prime}$, we have

$$
(\lambda I-\tilde{T}(\lambda))^{-1}=\left(\begin{array}{cc}
L(\lambda) & 0 \\
* & *
\end{array}\right)
$$

for suitable entries $*$;

4. $\tilde{T}(\lambda) \equiv \tilde{T}$ is independent of $\lambda$ if and only if $L(\lambda)$ is a left resolvent for $T$ on $\Omega$

5. If $\operatorname{dim} N(L(\lambda))<\infty$, then the map $\lambda \rightarrow \tilde{T}(\lambda)$ is analytic.

Theorem 3.3 follows from Theorem 3.4 and Theorem 3.5.

Using Lemma 2.1 and Theorem 3.3, we obtain the following consequence, improving part of Lemma 3.1.

Corollary 3.6. Let $T \in L(H)$ and let $\Omega \subset \bar{\Omega} \subset \rho_{\ell}(T) \cap \sigma(T)$ be an open, connected set. Then there exists an extension $\tilde{T}$ of $T$ on a larger Hilbert space $\tilde{H}=H \oplus H^{\prime}$ such that $R(\tilde{T})(\lambda)=(\lambda I-\tilde{T})^{-1}$ exists in $L(\tilde{H})$ for all $\lambda \in \Omega$ and $R(\tilde{T})(\lambda)\left(H^{\prime}\right) \subseteq H^{\prime}$, for all $\lambda \in \Omega$.

\subsection{Proof of Theorem 3.4. Let}

$$
\left(\begin{array}{ll}
L(\lambda) & S(\lambda) \\
T(\lambda) & V(\lambda)
\end{array}\right)
$$


be the matrix of $R(\lambda)=(\lambda I-\tilde{T})^{-1}$ with respect to the decomposition $\tilde{H}=H \oplus H^{\prime}$. Using the fact that $\tilde{T}$ is an extension of $T$ and the equality

$$
\left(\begin{array}{ll}
L(\lambda) & S(\lambda) \\
T(\lambda) & V(\lambda)
\end{array}\right)(\lambda I-\tilde{T})\left(\begin{array}{c}
h \\
0
\end{array}\right)=\left(\begin{array}{l}
h \\
0
\end{array}\right), \quad h \in H
$$

we obtain

$$
L(\lambda)(\lambda I-T) h=h
$$

for all $h \in H$. We identify $h \in H$ with $\left(\begin{array}{l}h \\ 0\end{array}\right) \in \tilde{H}$.

We have,

$$
\frac{L(\lambda)-L(\mu)}{\mu-\lambda}\left(\begin{array}{c}
h \\
0
\end{array}\right)=P_{H} \frac{R(\lambda)-R(\mu)}{\mu-\lambda}\left(\begin{array}{c}
h \\
0
\end{array}\right)=P_{H} R(\lambda) R(\mu)\left(\begin{array}{c}
h \\
0
\end{array}\right) .
$$

Therefore

$$
\left(\begin{array}{c}
\frac{L(\lambda) h-L(\mu) h}{\mu-\lambda} \\
0
\end{array}\right)=\left(\begin{array}{ll}
1 & 0 \\
0 & 0
\end{array}\right)\left(\begin{array}{cc}
L(\lambda) & S(\lambda) \\
T(\lambda) & V(\lambda)
\end{array}\right)\left(\begin{array}{cc}
L(\mu) & S(\mu) \\
T(\mu) & V(\mu)
\end{array}\right)\left(\begin{array}{c}
h \\
0
\end{array}\right)
$$

for all $h \in H$, yielding

$$
\frac{L(\lambda) h-L(\mu) h}{\mu-\lambda}=L(\lambda) L(\mu) h+S(\lambda) T(\mu) h
$$

for all $h \in H$.

Since $S(\lambda) T(\lambda)=0$, we obtain $L^{\prime}(\lambda)=-L(\lambda)^{2}$, for all $\lambda \in \Omega$. Then $L(\lambda)$ is a left resolvent (cf. for instance [7, Theorem 2.7]).

3.4. Proof of Theorem 3.5. Without loss of generality we may assume that $\Omega \subseteq \mathbb{D}$, the open unit disk.

Suppose Hilbert space $H^{\prime}$, operators $S, U(\lambda) \in L\left(H^{\prime}\right), \lambda \in \Omega$, function $\Omega \ni \lambda \rightarrow$ $K(\lambda) \in H^{\prime}$ and linear functional $r: H^{\prime} \rightarrow \mathbb{C}$ satisfying

1. $(\lambda I-S) U(\lambda)=I ; \lambda \in \Omega$,

2. $U(\lambda)(\lambda I-S)=I-r(\cdot) K(\lambda) ; \lambda \in \Omega$,

3. $r(K(\lambda))=1 ; \lambda \in \Omega$ (normalization)

are fixed. This set is nonvoid as the following example shows.

Example 3.7. Let $H^{\prime}=\ell^{2}$ and consider $S$ the backward shift operator, $U(\lambda)=$ $(\lambda U-I)^{-1} U, \quad \lambda \in \Omega$, where $U$ is the upward shift, $r(\cdot)=\left\langle\cdot, e_{1}\right\rangle$, where $e_{1}=$ $(1,0,0, \ldots), K(\lambda)=-(\lambda U-I)^{-1} e_{1}=\left(1, \lambda, \lambda^{2}, \lambda^{3}, \ldots\right)$ and $\langle\cdot, \cdot\rangle$ is the inner product in $\ell^{2}$. Then conditions 1$\left.)-3\right)$ above are satisfied.

Indeed, we have $S U=I$ and $U S=I-r(\cdot) e_{1}$, and thus

$$
(\lambda I-S) U(\lambda)=(\lambda I-S) U(\lambda U-I)^{-1}=I
$$

and

$$
\begin{aligned}
U(\lambda)(\lambda I-S) & =(\lambda U-I)^{-1} U(\lambda I-S) \\
& =(\lambda U-I)^{-1}\left\{\lambda U-I+r(\cdot) e_{1}\right\} \\
& =I+r(\cdot)(\lambda U-I)^{-1} e_{1} \\
& =I-r(\cdot) K(\lambda) .
\end{aligned}
$$

Condition 3) is easily verified.

The following properties will be useful: 
Properties 3.8. We have

a) $r(u)=0$ for all $u \in R(U(\lambda))$,

b) $(\lambda I-S) K(\lambda)=0$.

Here $R(T)$ denotes the range of $T$.

Proof. a). Condition 2) in the point $U(\lambda) h^{\prime}$ gives, using also the condition 1),

$$
U(\lambda) h^{\prime}=U(\lambda)(\lambda I-S) U(\lambda) h^{\prime}=U(\lambda) h^{\prime}-r\left(U(\lambda) h^{\prime}\right) K(\lambda) .
$$

Therefore $r\left(U(\lambda) h^{\prime}\right) K(\lambda)=0$. Using the normalization condition we obtain the desired relation $r\left(U(\lambda) h^{\prime}\right)=0$.

b). Using condition 2) and the normalization condition, we get

$$
U(\lambda)(\lambda I-S) K(\lambda)=K(\lambda)-K(\lambda)=0 .
$$

Since $U(\lambda)$ is left-invertible by condition 1 ), we obtain the desired conclusion.

To start off the proof of Theorem 3.5, we remark that $h-(\lambda I-T) L(\lambda) h \in$ $N(L(\lambda))$ for all $h \in H$. We obtain $H=R(\lambda I-T) \dot{+} N(L(\lambda))$, as an algebraic direct sum, for all $\lambda \in \Omega$. To prove the direct sum property, note that if $h=$ $(\lambda I-T) u \in N(L(\lambda))$, then

$$
u=L(\lambda)(\lambda I-T) u=L(\lambda) h=0
$$

and thus $h=0$.

It is easy to see that $\operatorname{dim} N(L(\lambda))$ is constant for all $\lambda \in \Omega$. We suppose in this part of the proof that $\operatorname{dim} N(L(\lambda))=\infty$ for all $\lambda \in \Omega$. If $\operatorname{dim} N(L(\lambda))=m<\infty$, the proof will be essentially the same (in fact even simpler since we have to deal with finite sums). Let $e_{j}(\lambda), j \geq 1$, be an orthonormal basis of $N(L(\lambda))$. Every $h \in H$ can be written as

$$
h=(\lambda I-T) L(\lambda) h+\sum_{j=1}^{\infty} c_{j}^{\lambda}(h) e_{j}(\lambda),
$$

where $c_{j}^{\lambda}(h) \in \mathbb{C}$. Since $L(\lambda)(\lambda I-T)=I$, we get

$$
(\lambda I-T) u=(\lambda I-T) u+\sum_{j=1}^{\infty} c_{j}^{\lambda}((\lambda I-T) u) e_{j}(\lambda) .
$$

Therefore $c_{j}^{\lambda}(h)=0$ if $h \in R(\lambda I-T)$. Since $e_{j}(\lambda) \in N(L(\lambda))$, it follows that $c_{j}^{\lambda}\left(e_{k}(\lambda)\right)=\delta_{j}^{k}$ for $j, k \geq 1$.

Construction of $\tilde{T}(\lambda)$. Consider $H^{\prime \prime}=H^{\prime} \oplus H^{\prime} \oplus \ldots$ the infinite direct sum of $H^{\prime}$ (if $m<\infty$, we can consider only the finite direct sum with $m$ times $H^{\prime}$ ). Set $\tilde{H}=H \oplus H^{\prime \prime}$. Let $\tilde{T}(\lambda) \in L(\tilde{H})$ be defined by

$$
\tilde{T}(\lambda)\left(h, h_{1}^{\prime}, h_{2}^{\prime}, \ldots\right)=\left(T h-\sum_{j=1}^{\infty} r\left(h_{j}^{\prime}\right) e_{j}(\lambda), S h_{1}^{\prime}, S h_{2}^{\prime}, \ldots\right) .
$$

Define also $\tilde{R}(\lambda) \in L(\tilde{H})$ by

$$
\tilde{R}(\lambda)\left(h, h_{1}^{\prime}, h_{2}^{\prime}, \ldots\right)=\left(L(\lambda) h, c_{1}^{\lambda}(h) K(\lambda)+U(\lambda) h_{1}^{\prime}, c_{2}^{\lambda}(h) K(\lambda)+U(\lambda) h_{2}^{\prime}, \ldots\right) .
$$

Proof of 1 . It can be easily seen that $\tilde{T}(\lambda)$ and $\tilde{R}(\lambda)$ are well defined. It is also easy to see that $\tilde{T}(\lambda)(h, 0,0, \ldots)=(T h, 0,0, \ldots)$ for all $h \in H$. Therefore $\tilde{T}(\lambda)$ is an extension of $T$. 
Proof of 2. We show now that $\tilde{R}(\lambda)=(\lambda I-\tilde{T}(\lambda))^{-1}$. Indeed, we have

$$
\begin{gathered}
\tilde{R}(\lambda)(\lambda I-\tilde{T}(\lambda))\left(h, h_{1}^{\prime}, \ldots\right) \\
=\tilde{R}(\lambda)\left((\lambda I-T) h+\sum_{j=1}^{\infty} r\left(h_{j}^{\prime}\right) e_{j}(\lambda),(\lambda I-S) h_{1}^{\prime}, \ldots\right) \\
=\left(L(\lambda)(\lambda I-T) h, r\left(h_{1}^{\prime}\right) K(\lambda)+U(\lambda)(\lambda I-S) h_{1}^{\prime}, \ldots\right)
\end{gathered}
$$

(since $L(\lambda) e_{j}(\lambda)=0$ and $c_{j}^{\lambda}(u)=0$ for all $u \in R(\lambda I-T)$ )

$$
=\left(h, h_{1}^{\prime}, \ldots\right) \text {. }
$$

On the other hand,

$$
\begin{gathered}
(\lambda I-\tilde{T}(\lambda)) \tilde{R}(\lambda)\left(h, h_{1}^{\prime}, \ldots\right) \\
=(\lambda I-\tilde{T}(\lambda))\left(L(\lambda) h, c_{1}^{\lambda}(h) K(\lambda)+U(\lambda) h_{1}^{\prime}, \ldots\right) \\
=\left((\lambda I-T) L(\lambda) h+\sum_{j=1}^{\infty} c_{j}^{\lambda}(h) e_{j}(\lambda),(\lambda I-S)\left(c_{1}^{\lambda}(h) K(\lambda)+U(\lambda) h_{1}^{\prime}\right), \ldots\right) \\
\quad(\text { using Property a }) \\
=\left(h,(\lambda I-S) U(\lambda) h_{1}^{\prime}, \ldots\right) \\
(\text { using Property b) } \\
=\left(h, h_{1}^{\prime}, \ldots\right) .
\end{gathered}
$$

Thus $\tilde{R}(\lambda)=(\lambda I-\tilde{T}(\lambda))^{-1}$ and Property 2 in the theorem is proved.

Proof of 3. It is clear from the definition that, with respect to the decomposition $\tilde{H}=H \oplus H^{\prime \prime}$, we have

$$
(\lambda I-\tilde{T}(\lambda))^{-1}=\tilde{R}(\lambda)=\left(\begin{array}{cc}
L(\lambda) & 0 \\
* & *
\end{array}\right)
$$

Proof of 4. The fourth property $(L(\lambda)$ is a left resolvent iff $\tilde{T}(\lambda)$ is independent of $\lambda$ ) follows from the definition of $\tilde{T}(\lambda)$ and the fact that a left-inverse function $L(\lambda)$ is a left resolvent if and only if the kernel $N(L(\lambda)$ ) is independent of $\lambda$ (cf. [6, Lemma 9.6]).

Proof of 5. Suppose, for the last property, that $m<\infty$. According to a result of [15] and [8, page 187], the orthonormal basis $e_{j}(\lambda), j=1, \ldots, m$, can be chosen such that $\lambda \rightarrow e_{j}(\lambda)$ is analytic. This implies that the map $\lambda \rightarrow \tilde{T}(\lambda)$ is analytic. 


\section{REFERENCES}

1. G.R. Allan : On one-sided inverses in Banach algebras of holomorphic vector-valued functions, J. London Math. Soc. 42 (1967), 463-470. MR 35:5939

2. G.R. Allan : Holomorphic vector-valued functions on a domain of holomorphy, J. London Math. Soc. 42 (1967), 509-513. MR 35:5940

3. C. Apostol : The correction by compact perturbations of the singular behaviour of operators, Rev. Roumaine Math. Pures et Appl. 21 (1976), 155-175. MR 58:7180

4. C. Apostol, K. Clancey : On generalized resolvents, Proc. Amer. Math. Soc. 58 (1976), 163168. MR 53:14162

5. C. Apostol, K. Clancey : Generalized inverses and spectral theory, Trans. Amer. Math. Soc. 215 (1976), 293-300. MR 52:4002

6. C. Apostol, L.A. Fialkow, D.A. Herrero, D. Voiculescu : Approximation of Hilbert space Operators, Vol. 2, Pitman Res. Notes in Math. 102, 1984. MR 85m:47002

7. C. Badea, M. Mbekhta : Generalized inverses and the maximal radius of regularity of a Fredholm operator, Integral Eq. Oper. Th. 28 (1997), 133-146. MR 98e:47018

8. H. Bart: Holomorphic relative inverses of operator valued functions, Math. Ann. 208 (1974), 179-194. MR 49:11289

9. D.A. Herrero : Approximation of Hilbert space Operators, Vol. 1, 2nd ed, Pitman Res. Notes in Math. 224, 1989. MR 91k:47002

10. J.-Ph. Labrousse, M. Mbekhta :Résolvant généralisé et séparation des points singuliers quasiFredholm, Trans. Amer. Math. Soc. 333 (1992), 299-313. MR 92k:47007

11. M. Mbekhta : Généralisation de la décomposition de Kato aux opérateurs paranormaux et spectraux, Glasgow Math. J. 29 (1987), 159-175. MR 88i:47010

12. M. Mbekhta : Résolvant généralisé et théorie spectrale, J. Operator Th. 21 (1989), 69-105. MR 91a:47004

13. T.J. Ransford : Generalised spectra and analytic multivalued functions, J. London Math.Soc. 29 (1984), 306-322. MR 85f:46091

14. C.J. Read : Extending an operator from a Hilbert space to a larger Hilbert space so as to reduce its spectrum, Israel J. Math. 57 (1987), 375-380. MR 88e:47014

15. P. Saphar : Contribution a l'étude des applications linéaires dans un espace de Banach, Bull. Soc. Math. France 92 (1964), 363-384. MR 32:4549

16. P. Saphar : Sur les applications linéaires dans un espace de Banach II, Ann. Sci. Ecole Norm. Sup. Ser. 3, 82 (1965), 205-240. MR 35:2164

17. M.A. Shubin : On holomorphic families of subspaces of a Banach space, Integral Eq. Oper. Th. 2-3 (1979), 407-420. MR 80m:46045

18. Z. Słodkowski : Analytic set-valued functions and spectra, Math. Ann. 256 (1981), 363-386. MR 83b: 46070

19. J. Zemánek : One-sided spectral calculus, Problem 2.10 in : Linear and Complex Analysis Problem Book 3 (V.P. Havin, N.K. Nikolski, eds.), Lect. Notes Math. 1573 (1994), 102-104. MR 96c:00001a

URA 751 au CNRS \& UFR de Mathématiques, Université de Lille I, F-59655 VilLENEUVE D'AscQ, France

E-mail address: badea@gat.univ-lille1.fr

Current address, M. Mbekhta: University of Galatasaray, Çiragan Cad no 102, Ortakoy 80840, Istanbul, Turkey

E-mail address: mbekhta@gat.univ-lille1.fr 